\title{
CX3CL1(+) Microparticles Mediate the Chemoattraction of Alveolar Macrophages toward Apoptotic Acute Promyelocytic Leukemic Cells
}

\author{
Wen-Hui Tsai ${ }^{a}$ Chung-Hung Shih ${ }^{a}$ Shan-Yu Feng ${ }^{b}$ I-Ting Li ${ }^{b}$ Shao-Chi Chang ${ }^{b}$ \\ Yu-Chieh Lin ${ }^{b}$ Hui-Chi Hsub,c,d \\ aDepartment of Respiratory Therapy, Taipei Medical University, ${ }^{b}$ Department of Physiology and \\ 'Department of Medicine, School of Medicine, National Yang-Ming University, ${ }^{\mathrm{d}}$ Division of General \\ Medicine, Department of Medicine, Taipei-Veterans General Hospital, Taipei, Taiwan
}

\section{Key Words}

Apoptosis - Acute lung injury - Acute promyelocytic leukemia - Chemotaxis - CX3CL1 • CX3CR1 • Differentiation syndrome $\bullet$ Fractalkine $\bullet$ Phagocytosis $\bullet$ Microparticles $\bullet$ Resolution

\begin{abstract}
Background/Aims: During the resolution phase of inflammation, release of "find-me" signals by apoptotic cells is crucial in the chemoattraction of macrophages toward apoptotic cells for subsequent phagocytosis, in which microparticles derived from apoptotic cells (apo-MPs) are involved. A recent study reports that CX3CL1 is released from apoptotic cells to stimulate macrophages chemotaxis. In this study, we investigated the role of CX3CL1 in the apo-MPs in the cell-cell interaction between alveolar macrophage NR8383 cells and apoptotic all-trans retinoic acid-treated NB4 (ATRA-NB4) cells. Methods/Results: Apoptotic ATRA-NB4 cells and their conditioning medium (CM) enhanced the chemoattraction of NR8383 cells as well as their phagocytosis activity in engulfing apoptotic ATRA-NB4 cells. The levels of $\mathrm{CX} 3 \mathrm{CL} 1(+)$ apo-MPs and CX3CL1 were rapidly elevated in the CM of ATRA-NB4 cell culture after induction of apoptosis. Both exogenous CX3CL1 and apo-MPs enhanced the transmigration of NR8383 cells toward apoptotic ATRA-NB4 cells. This pro-transmigratory activity was able to be partially inhibited either by blocking the CX3CR1 (CX3CL1 receptor) of NR8383 cells with its specific antibody or by blocking the surface CX3CL1 of apo-MPs with its specific antibody before incubating these apo-MPs with NR8383 cells. Conclusion: CX3CL1(+) apo-MPs released by apoptotic cells mediate the chemotactic transmigration of alveolar macrophages.
\end{abstract}

Copyright (c) 2014 S. Karger AG, Basel

Hui-Chi Hsu, MD,

and Chung-Hung Shih, MD, PhD.
Division of General Medicine, Department of Medicine, Taipei Veterans General Hospital, 201, Sec. 2, Shih-Pai Rd, Taipei, 11217 (Taiwan) and Department of Respiratory Therapy, Taipei Medical University 250 Wu-Hsing Street, Taipei 11052 (Taiwan)

E-Mail hchsu@vghtpe.gov.tw and E-Mail chshih43@tmu.edu.tw 
Tsai et al.: CX3CL1(+) Microparticles Stimulate Alveolar M $\phi$ Chemotaxis

\section{Introduction}

All-trans retinoic acid (ATRA) has been used successfully in the treatment of acute promyelocytic leukemia (APL) by inducing APL cell differentiation into mature granulocytes. However, this treatment can be complicated by the occurrence of differentiation syndrome (DS) in $25 \%$ of APL patients [1]. DS has the full clinical manifestations associated with acute lung injury including fever, dyspnea, hypoxemia, a diffuse pulmonary infiltrate detectable on chest x-ray and a massive infiltration of APL cells into the alveolar spaces [1]. Previous works have shown that the granulocytic differentiation and functional activation in ATRAtreated APL (ATRA-APL) cells contribute to the transmigration of circulating ATRA-APL cells into alveolar spaces, resulting in the development of acute lung injury in DS patients. Several chemokines are involved in the pathogenesis of DS, such as interleukin-8 and monocyte chemotactic protein -1 [1-3]. Recently, we reported that the alveolar macrophage $(\mathrm{AM} \phi)$ is able to engulf apoptotic ATRA-APL cells; this may contribute to the clearance of apoptotic APL cells in the alveolar space during the resolution phase of DS [4]. However, the mechanism underlying the chemoattraction of $\mathrm{AM} \phi$ toward apoptotic APL cells has not been investigated in detail.

$\mathrm{AM} \phi$ is strategically located in the alveolar space and functions primarily against invading pathogens, microorganisms, or environmental toxins [5]. During the resolution phase of acute lung injury, $\mathrm{AM} \phi$ are able to undertake phagocytosis of apoptotic leukocytes, contributing to the clearance of leukocytes from the lung and the restoration of epithelial barrier function [5]. Before the tethering/engulfment phases of $\mathrm{AM} \phi$-mediated clearance of apoptotic cells, $\mathrm{AM} \phi$ are required to navigate effectively to sites of apoptotic cells. Regarding this, the apoptotic cells are able to release many "find-me" signals such as ribosomal protein S19, endothelial monocyte-activating polypeptide II (EMAPII), and lysophosphatidylcholine, at an early apoptotic stage to chemoattract $\mathrm{AM} \phi$ for subsequent phagocytosis [6, 7]. Recent studies have reported that microparticles (MPs) released from apoptotic cells play a role in navigating mononuclear phagocytes toward apoptotic cells for subsequent phagocytosis [8, 9].

CX3CL1, also known as fractalkine, is a chemokine and intercellular adhesion molecule. CX3CL1 is a type I transmembrane protein, the extracellular portion of which comprises a chemokine domain attached to a mucin stalk [10]. The membrane-bound full-length surface CX3CL1 functions as an adhesion molecule and enables the integrin-independent capture and firm adhesion of leukocytes via the CX3CL1 receptor, which is called CX3CR1 [10]. Under inflammatory status, the extracellular chemokine domain of CX3CL1 can be cleaved/shed by a disintegrin and metalloproteinase domain (ADAM) to generate a soluble chemokine from stimulus-exposed cells [10]. Recently, Truman et al. reported that CX3CL1 is released from apoptotic lymphocytes to stimulate the macrophage's chemotactic transmigration, and a part of CX3CL1 is in association with MPs released by those apoptotic lymphocytes [9]. However, whether CX3CL1 mediates the chemoattractant property of MPs derived from apoptotic cells (apo-MPs) has been rarely studied.

Based on this evidence, it is logical to test the hypothesis that apoptotic cells release CX3CL1(+) apo-MPs to stimulate the chemoattraction of macrophages toward apoptotic cells. In the present study, we used apoptotic ATRA-treated NB4 (ATRA-NB4) cells to represent apoptotic ATRA-APL cells in the alveolar spaces among patients with DS. Using an in vitro cell co-culture model, we investigated the role of $\operatorname{CX3CL(+)~apo-MPs~in~the~cell-cell~}$ interactions between $\mathrm{AM} \phi$ (NR8383) cells and apoptotic ATRA-NB4 cells.

\section{Materials and Methods}

Cell culture and the preparation of conditioned medium (CM)

NB4 cells are a human APL cell line (a gift from M. Lanotte, INSERM U-301, SDI No 15954.1 CNRS, Centre Hayem, Paris, France ). NR8383 cells (CRL-2192; ATTC, Manassas, VA) are a well-characterized, semi- 
Tsai et al.: CX3CL1(+) Microparticles Stimulate Alveolar M $\phi$ Chemotaxis

adherent $\mathrm{AM} \phi$ cell line that is derived from normal Sprague-Dawley (SD) rat lung and can be extrapolated to freshly derived AM $\phi$ of various species[11]. Both NB4 cells and NR8383 cells were cultured in RPMI1640 medium (GIBCO, Grand Island, NY) and in Ham's F12K medium, respectively, as described previously [4]. ATRA treatment can induce NB4 cells into the process of granulocytic differentiation [1]. NB4 cells $\left(1 \times 10^{5}\right.$ cells $\left./ \mathrm{ml}\right)$ were treated with ATRA $(1 \mu \mathrm{M}$; Sigma, St. Louis, MO, USA] for 3 days before receiving UV irradiation. ATRA-NB4 cells and NR8383 cells were adjusted to the cell densities of $1 \times 10^{6} \mathrm{cells} / \mathrm{ml}$ and $2 \times 10^{6}$ cells $/ \mathrm{ml}$, respectively, before further experiments.

To prepare CM, supernatants from ATRA-NB4 cell cultures, both before and after UV irradiation, were harvested and then centrifuged at $250 \mathrm{~g}$ for 5 minutes to remove all cellular components. Finally, these CMs either underwent MP purification or were stored as aliquots at $-20^{\circ} \mathrm{C}$.

\section{Generation of apoptotic cells}

To induce apoptosis, ATRA-NB4 cells were irradiated with $254 \mathrm{~nm}$ wavelength UV light $\left(2000 \mathrm{~mJ} / \mathrm{cm}^{2}\right)$ using a UV lamp (Spectroline; Westbury, NY) [12]. Subsequent to the irradiation, the cells were incubated for up to 24 hours at $37^{\circ} \mathrm{C}$. Apoptosis was detected by double-staining the washed UV-treated ATRA-NB4 (UV-ATRA-NB4) cells with annexin V (NXPE; R\&D Systems, Minneapolis, USA) and 7-amino-Actinomycin-D (7-AAD; BD Bioscience Pharmingen, San Diego, USA), which allows early apoptotic cells to be distinguished from late apoptotic cells by flow cytometry analysis [13].

\section{MPs preparation and flow cytometry analysis}

MPs from UV-ATRA-NB4 cell cultures were prepared as reported by Gasser et al.[14]. The CM of ATRANB4 cell culture (ATRA $1 \mu \mathrm{M}$ for 3 days) was collected and centrifuged at $1,500 \mathrm{~g}$ for 5 minutes prior to further ultracentrifugation at 100,000 g for 1 hour $\left(0\right.$ ptima $^{\mathrm{TM}} \mathrm{L}-100 \mathrm{XP}$ Ultracentrifuge; Beckman Coulter, Fullerton, California, USA). The MP pellet was washed once and re-suspended in PBS. For flow cytometry analysis, the MPs were stained with annexin V (NXPE; R\&D Systems, Minneapolis, USA) or anti-CX3CL1 (MAB365; R\&D System, Minneapolis, USA) antibodies. The flow-cytometric analysis of the MP preparations showed the expected heterogeneous populations, with sizes varying approximately between 0.1 and $2 \mu \mathrm{m}$ as verified by the use of control beads (2- $\mu$ m each; ACBP-20-10; Becton Dickson). The numbers of MPs within the same gated area of MPs in the flow cytometry dot plots, as shown in Figure 3A, were then calculated $[15,16]$.

\section{Flow cytometry analysis of ATRA-NB4 cells and NR8383 cells}

The non-permeablizied ATRA-NB4 cells and NR8383 cells were stained with either rabbit CX3CL1 polyclonal antibody (MAB365; R\&D System, Minneapolis, USA) and rabbit CX3CR1 polyclonal antibody (AF5825; R\&D System, Minneapolis, USA), respectively, before analysis by FAC Scan as reported previously $[4,15]$.

\section{Transmigration assay}

The transmigration assay was performed in a co-culture system (BD Falcon ${ }^{\mathrm{TM}}$, San Jose, CA) using an upper insert with membrane ( $8 \mu \mathrm{m}$ pore size, $1 \times 10^{5}$ pores $/ \mathrm{cm}^{2}$ ) [2]. NR8383 cells were transferred to the upper insert of the transmigration assay system, while either apoptotic ATRA-NB4 cells or their CMs were added into the lower plate. The upper insert and lower plate were assembled and incubated for 2 hours. The diagram of transmigration assay has been demonstrated in our previous reports [2, 3]. Transmigration cell numbers were calculated as described previously [2]. The results were expressed as a migration index indicating a fold increase relative to the number of NR8383 cells transmigrating toward either apoptotic cells or their CM.

\section{Phagocytosis of apoptotic NB4 cells}

NR8383 cells were cultured in plates at $37^{\circ} \mathrm{C}$ for 3 days before being co-cultured with either UV-untreated or UV-treated ATRA-NB4 cells $\left(2 \times 10^{6}\right.$ cells/well $)$ at $37^{\circ} \mathrm{C}$ for 2 hours before assaying for phagocytosis. Non-ingested cells were removed after washing with PBS. After myeloperoxidase staining, the number of NR8383 cells containing ingested NB4 cells was counted by light microscopy [4]. The results were expressed as phagocytosis index indicating a fold increase relative to the percentage of NR8383 cells engulfing UV-untreated ATRA-NB4 cells as control. 
Phagocytosis of florescence-labeled apoptotic cells

The fluorescent membrane stains Alexa Fluor 488 phalloidin (green; Invitrogen, Oregon, OR, USA) and PKH-26 (red; Sigma-Aldrich, St. Louis, MO, USA) were used to label NR8383 cells and apoptotic UVATRA-NB4 cells, respectively. Thereafter, the labeled NR8383 cells were co-cultured with labeled apoptotic UV-ATRA-NB4 cells $\left(2 \times 10^{6}\right.$ cells $)$ at $37^{\circ} \mathrm{C}$ for 2 hours before being photographed under a fluorescent microscope [12].

Western blotting

ATRA-NB4 cells were irradiated and then incubated for up to 24 hours at $37^{\circ} \mathrm{C}$ before performing protein extraction and Western blots as described previously [15]. Mouse polyclonal antibodies against human CX3CL1 (MAB3561; R\&D Systems, Minneapolis, USA) and monoclonal antibody against Glyceraldehyde-3phosphate dehydrogenase (GAPDH) (Sigma, St. Louis, Missouri, USA) were used for the Western blotting. The results were first normalized against the GAPDH present in the same cells and then expressed as a fold increase relative to the UV un-irradiated ATRA-NB4 cells as control $[4,15]$.

Measurement of CX3CL1

The level of CX3CL1 in the CM was determined using an enzyme-linked immunosorbent assay (ELISA) kit (R\&D Systems; Minneapolis, MN, USA) following the manufacturer protocol [17, 18].

\section{Statistical analysis}

The results were evaluated by one way ANOVA followed by the Fisher's least significant difference (LSD) procedure where appropriate. A value of $p<0.05$ was considered significant. All results are presented as mean $\pm \mathrm{SD}$.

\section{Results}

NR8383 cells chemoattractants are produced by apoptotic ATRA-NB4 cells

We first determined the effect of UV irradiation on the induction of apoptosis in ATRANB4 cells at 4-hours, 16-hours and 24-hours after UV irradiation. Table 1 demonstrates that, after UV irradiation, the percentage of early apoptotic ATRA-NB4 cells significantly increased at 4-hour after UV irradiation $(\mathrm{p}<0.001)$, and the percentage of both early and late apoptotic cells also increased significantly in a time-dependent manner $(p<0.001 \& p<0.001$ : respectively).

We then determined the transmigratory activity of NR8383 cells toward apoptotic ATRA-NB4 cells which had been induced into the process of apoptosis by UV-irradiation at 4 to 24 hours previously. Figure 1A demonstrates that the transmigratory activity of NR8383 cells toward apoptotic ATRA-NB4 cells was significantly higher than those cells toward UVuntreated ATRA-NB4 cells; and this transmigratory activity increased in a time-dependent manner when the duration after irradiation of the apoptotic ATRA-NB4 cells was increased $(p<0.001)$.

Thereafter, we determined the phagocytosis activity of NR8383 cells on engulfing apoptotic ATRA-NB4 cells. Consistent with our previous study [4], Figure 1(B-E) demonstrates that NR8383 cells had a higher phagocytic activity in engulfing apoptotic ATRA-NB4 cells than in engulfing living ATRA-NB4 cells; and that the phagocytic activity increased in a timedependent manner as the time after UV irradiation of the co-cultured apoptotic ATRA-NB4 cells increased $(p<0.001)$.

It now became important to determine whether chemo-attractants were released by the apoptotic ATRA-NB4 cells after triggering of the apoptotic program. CMs, harvested from apoptotic ATRA-NB4 cell cultures, were added into the lower plates of the transmigratory system to determine their chemotactic activity. Figure $2 \mathrm{~A}$ demonstrates that $\mathrm{CM}$ of apoptotic ATRA-NB4 cell culture harvested at 4 hours after UV irradiation had an enhanced chemoattractant activity on NR8383 cells than CM harvested from UV-untreated cells $(p<0.001)$, and this activity continued to increase gradually up to 24 hours after UV irradiation. 


\begin{tabular}{ccc}
\hline Hours after UV Tx & Early apoptosis (\%) & Late apoptosis (\%)+ \\
\hline 0 & $0.5 \pm 0.2$ & $1.4 \pm 0.4$ \\
4 & $11.2 \pm 2.5^{\#}$ & $10.0 \pm 2.4$ \\
18 & $13.3 \pm 1.7^{\#}$ & $33.2 \pm 6.5^{\# \#, *}$ \\
24 & $12.1 \pm 1.0^{\#}$ & $42.0 \pm 14.0^{\# \#, * *}$ \\
& & \\
$p$ value $^{++}$ & $p<0.001$ & $p<0.001$ \\
\hline
\end{tabular}

Table 1. Ultra-violet irradiation induced apoptosis in the ATRA-NB4 cells. +Early apoptosis is defined as cells expressing annexin V, but not 7-AAD; and late apoptosis: defined as cells expressing both annexin $\mathrm{V}$ and 7-AAD, ${ }^{++}$: ANOVA; \#: $p<0.001$ vs. 0 -hour (early apoptosis); \#\#: $p<0.001$ vs. 0 -hour (late apoptosis); *: $p<0.05$ vs. 4-hour (late apoptosis); **: $p<0.001$ vs. 4-hour (late apoptosis)

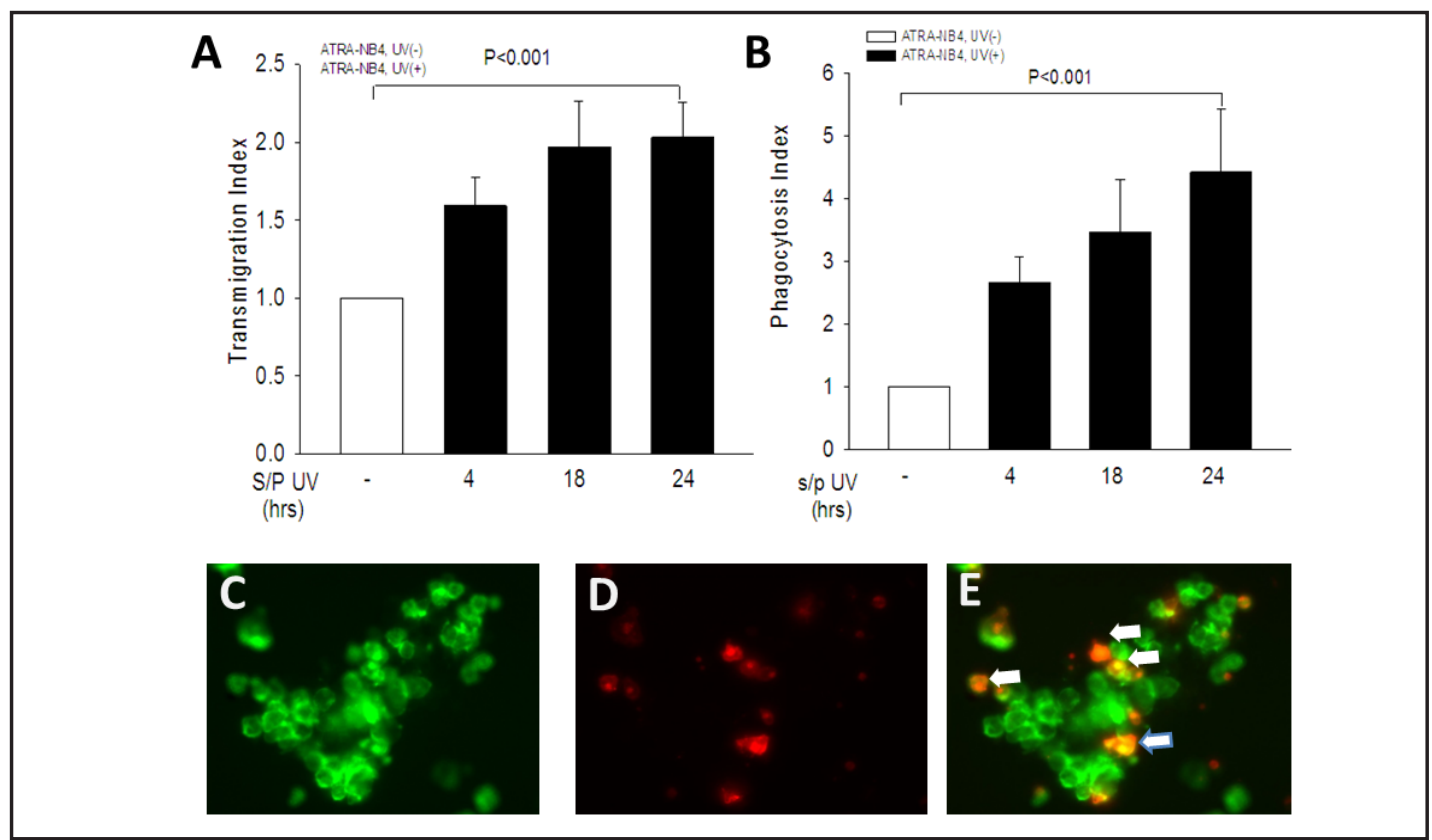

Fig. 1. Transmigration of NR8383 cells toward apoptotic ATRA-NB4 cells for subsequent phagocytosis. (A) NR8383 cells were added into the upper insert of transmigratory assay system, and UV-unirradiated ATRANB4 cells (blank bar) or UV-irradiated ATRA-NB4 cells (4-24 hours after UV; black bar) were added into the lower plates. The upper insert and lower plate were assembled to determine the chemotactic activity (Materials and Methods section). The results were expressed as migration index indicating a fold increase relative to the number of NR8383 cells transmigrating toward UV-untreated ATRA-NB4 cells as control. (B) NR8383 cells were cultured in plates for 3 days before UV-ATRA-NB4 cells were added into such plates for phagocytosis (Materials and Methods). The results were expressed as phagocytosis index indicating a fold increase relative to the percentage of NR8383 cells engulfing UV-untreated ATRA-NB4 cells as control. The results are presented as the means \pm SD from six (A) and four (B) independent experiments, respectively. The results were analyzed by ANOVA. (C)The Alexa Fluor 488 phalloidin-labeled NR8383 cells (green) and (D) PKH26-labeled apoptotic UV-ATRA-NB4 cells (red) were co-cultured for 2 hours before being photographed by fluorescent microscope. (E) Overlay of photograph C and D. White arrows indicate apoptotic ATRA-NB4 cells were engulfed by NR8383 cells (yellow). Magnification x 300.

We then examined whether CX3CL1 was released into the CMs by apoptotic ATRA-NB4 cells. Figure 2B demonstrates that CX3CL1 was constitutively released by ATRA-NB4 cells; the level of CX3CL1 was significantly increased 4 hours after induction of apoptosis by UV irradiation $(p<0.05)$, and this level remained persistently elevated thereafter. 

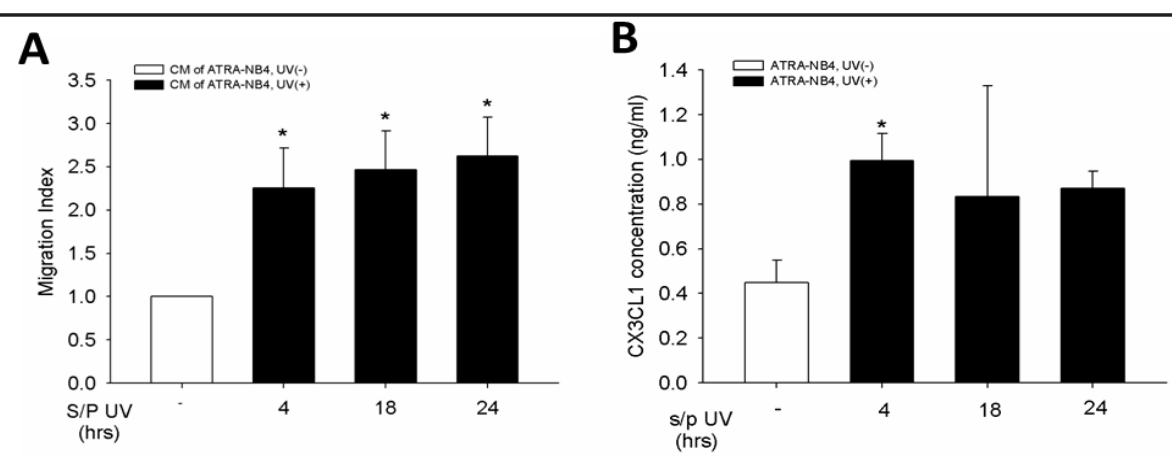

Fig. 2. Chemotactic activity and the level of CX3CL1 in the conditioning medium harvested from apoptotic ATRA-NB4 cell cultures. (A) The CMs were harvested from UV-ATRA-NB4 cell cultures, either before (blank bar) or 4-24 hours after (black bar) UV irradiation, which were added into the lower plate of transmigratory assay system, while NR8383 cells were added separately on the upper insert. The upper insert and lower plate were assembled to determine the chemotactic activity (Materials and Methods section). The results were expressed as migration index indicating a fold increase relative to the number of NR8383 cells transmigrating toward CM harvested from UV-untreated ATRA-NB4 cell culture as control. *: $p<0.001$ vs. CM harvested from UV-untreated ATRA-NB4 cell culture; (B) The level of CX3CL1 in the CMs was determined by ELISA. * $p<0.05$ vs. UV-untreated ATRA-NB4 cells. The results are presented as the means \pm SD from six (A) and five (B) independent experiments, respectively.

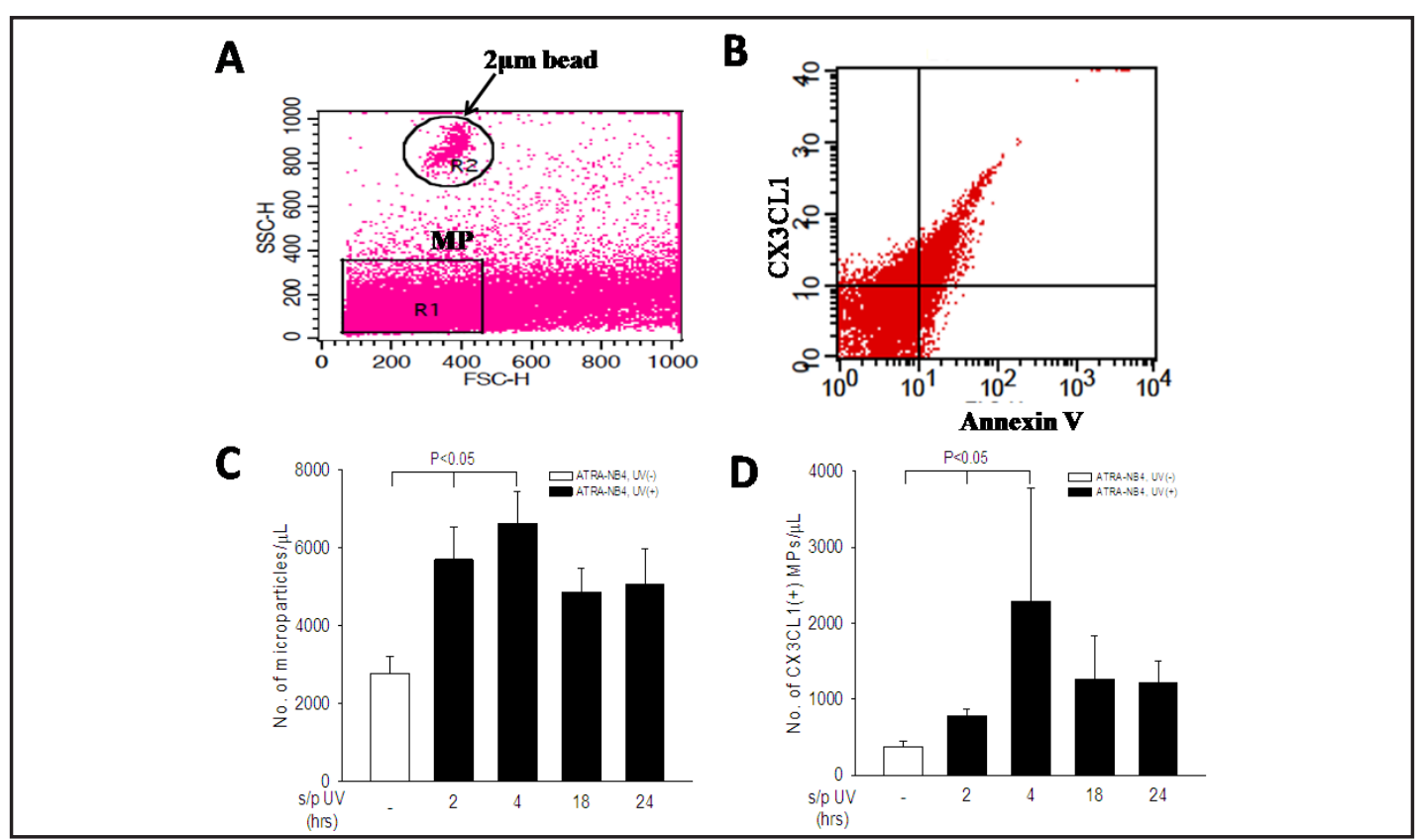

Fig. 3. Flow cytometric analysis of MPs released from apoptotic ATRA-NB4 cells. MPs were purified from CM of ATRA-NB4 cell cultures at either before or 2-24 hours after UV irradiation. (A) Dot plot showing the heterogeneity in MPs size; circled dots refer to $2 \mu \mathrm{m}$ beads run in parallel as internal control. (B) Expression of CX3CL1 and annexin V on MPs in the flow cytometric analysis was determined by using antibody specific for CX3CL1 and annexin V. The total numbers of apo-MPs (C) and CX3CL1(+) apo-MPs (D), in the $20 \mathrm{ml} \mathrm{CM}$ harvested from UV-treated ATRA-NB4 cell cultures, were calculated using a known concentration of flow count beads. These represent the means \pm SD from five independent experiments $(C, D)$.

\section{CX3CL1(+) apo-MPs are released by apoptotic ATRA-NB4 cells}

We subsequently purified apo-MPs from the CMs of apoptotic ATRA-NB4 cell cultures. Figure 3 demonstrates that, as compared with MPs released by living cells, the level of apoMPs released by apoptotic ATRA-NB4 cells increased progressively over the first 4 hours 


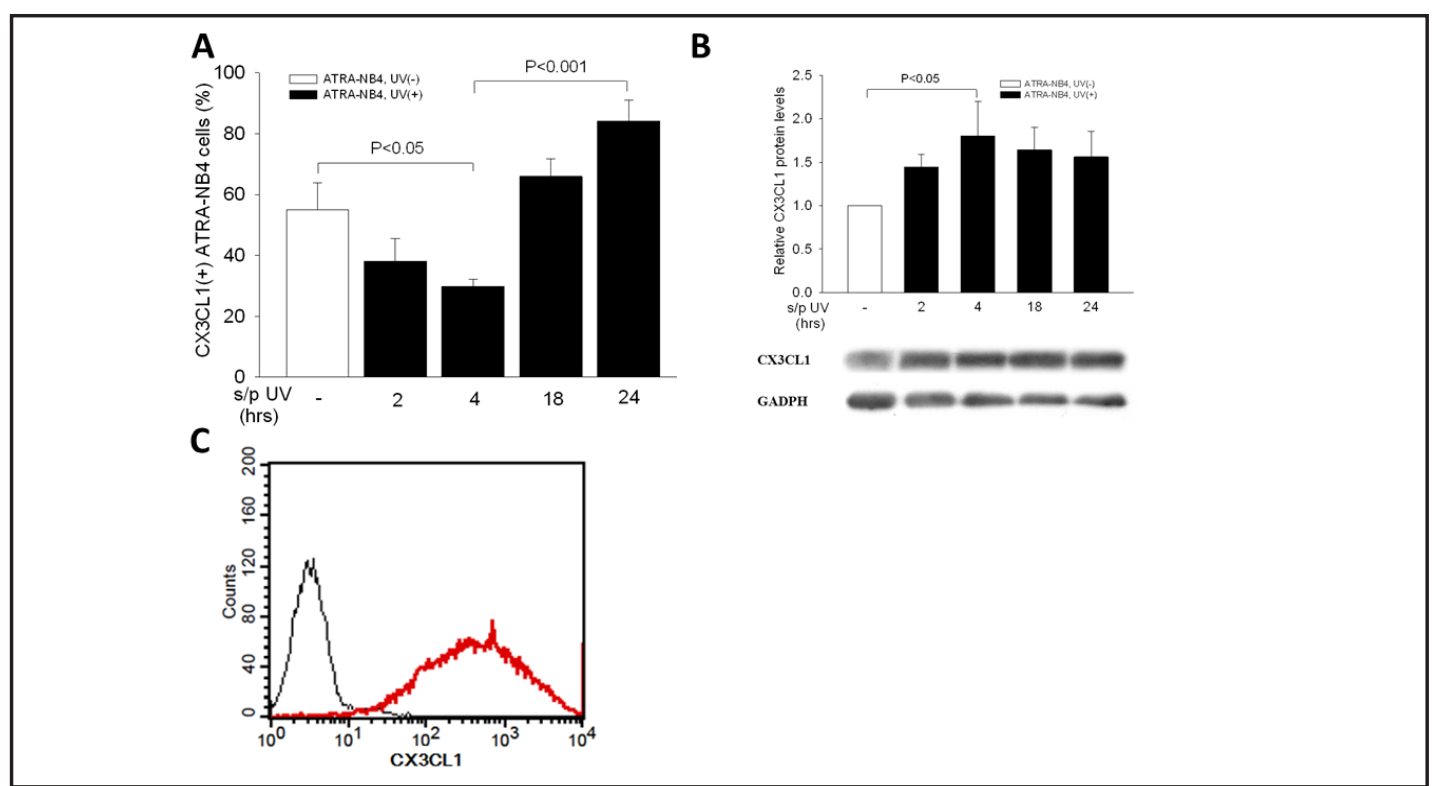

Fig. 4. Characterization of CX3CL1 expression on ATRA-NB4 cells by flow cytometry and western blotting, and CX3CR1 expression on NR8383 cells by flow cytometry. (A) ATRA-NB4 cells were first treated with UV-irradiation 2-24 hours before determining their surface expression of CX3CL1 by flow cytometric analysis. The results are presented as the means \pm SD from six independent experiments, and were analyzed by ANOVA. (B) The levels of CX3CL1 protein in the whole cells of ATRA-NB4 cells were determined by western blotting. The results were first normalized against GAPDH from the same cells and subsequently expressed as a fold increase relative to the UV-untreated ATRA-NB4 cells. The results are presented as the means \pm SD from five independent experiments, and were analyzed by ANOVA. (C) Surface expression of CX3CR1 on the NR8383 cells as determined by flow cytometric analysis. This is a representative picture from four independent experiments.

after induction of apoptosis by UV-treatment $(p<0.05)$, and thereafter this level decreased gradually until the 24-hour point after UV irradiation. Both CX3CL1 and annexin V were expressed on the surface of these apo-MPs (Fig. 3B); and the level of CX3CL1(+) apo-MPs in the CMs of apoptotic ATRA-NB4 cell cultures was also significantly elevated at 4-hours after UV irradiation than those MPs released by the living cells $(p<0.05$; Fig. 3D).

Expression of CX3CL1 on UV-ATRA-NB4 cells and the expression of CX3CR1 on NR8383 cells

Further flow cytometry analysis of the surface expression of apoptotic ATRA-NB4 cells indicated that CX3CL1 was expressed on the UV-untreated ATRA-NB4 cells, and that its level initially decreased at 4-hours after induction of apoptosis by UV irradiation. However, this level then increased when measured at 18- and 24-hours after UV irradiation (Fig. 4A). We also determined the protein level of CX3CL1 in the apoptotic ATRA-NB4 cells by the Western blotting assay. Figure 4B shows that, as compared with UV-untreated cells, the level of CX3CL1 protein was found to increase progressively in the apoptotic ATRA-NB4 cells over the first 4 hours after UV irradiation $(p<0.05)$, but then underwent no significant change thereafter up to the end of the UV irradiation study.

Flow cytometry analysis also demonstrated that CX3CR1 was expressed on the surface of NR8383 cells (Fig. 4C).

CX3CL1 mediates the pro-transmigratory property of CX3CL1(+) apo-MPs released by apoptotic-ATRA-NB4 cells

Figure 5A demonstrates that transmigratory activity of NR8383 cells toward apoptotic ATRA-NB4 cells was inhibited when the CX3CR1 of the NR8383 cells were previously blocked 


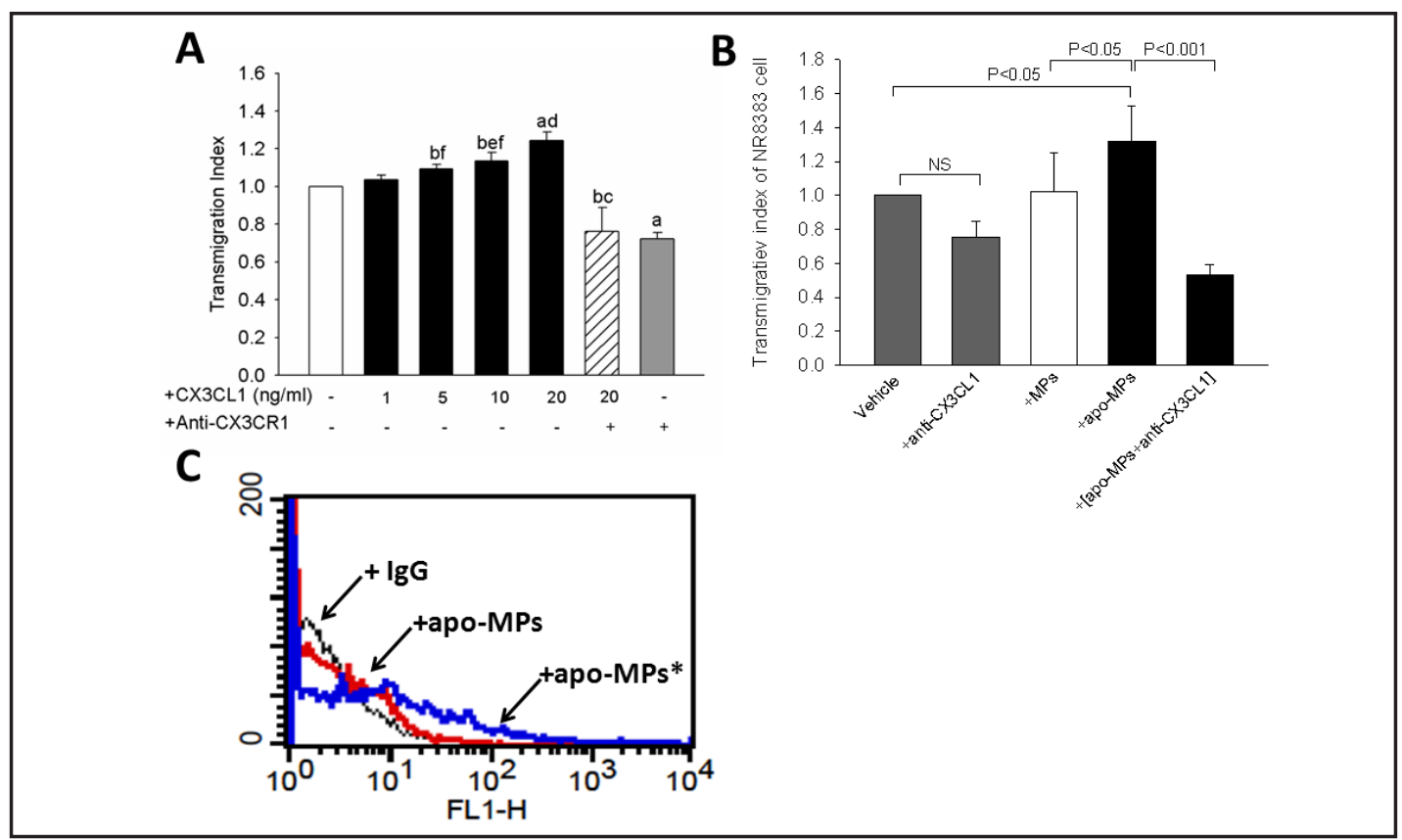

Fig. 5. CX3CL1 mediates the pro-transmigratory property of CX3CL1(+) apo-MPs released by apoptoticATRA-NB4 cells. (A) NR8383 cells were treated with exogenous CX3CL1 (1-20 ng/ml; black bar) before being added to the upper insert of the transmigratory assay system. Part of NR8383 cells were either treated with anti-CX3CR1 antibody (grey bar) or treated with anti-CX3CR1 antibody followed by exogenous CX3CL1 treatment (striped bar). The results were expressed as migration index indicating a fold increase relative to the number of NR8383 cells transmigrating toward UV-untreated ATRA-NB4 cells in the absence of antiCX3CR1 antibody and exogenous CX3CL1 treatment (blank bar). a: $P<0.001$ vs. blank bar; b:P<0.05 vs. blank bar; c: P<0.001 vs. CX3CL1 $20 \mathrm{ng} / \mathrm{ml}$ only; d: P<0.001 vs. CX3CL1 $1 \mathrm{ng} / \mathrm{ml}$ only; e: P<0.05 vs. CX3CL1 $1 \mathrm{ng} /$ $\mathrm{ml}$ only; f: $\mathrm{P}<0.05$ vs. CX3CL1 $20 \mathrm{ng} / \mathrm{ml}$ only (B) NR8383 cells were firstly incubated with vehicle (grey bar), anti-CX3CL1 (grey bar), MPs derived from UV un-irradiated ATRA-NB4 cell cultures(+MPs; blank bar) , apo-MPs derived from apoptotic ATRA-NB4 cell cultures (+apo-MPs; black bar) or apo-MPs that had been pretreated with antibody specific against CX3CL1 (+apo-MPs + anti-CX3CL1; black bar), respectively, before being added to the upper insert of the transmigratory assay system. The apoptotic ATRA-NB4 cells (4 hours after UV irradiation) were added into the lower plate (A \& B). Upper insert and lower plate were assembled for migratory assay (Materials and Methods section). The results were expressed as migration index indicating a fold increase relative to the number of vehicle-treated NR8383 cells transmigrating toward UV-untreated ATRA-NB4 cells. The results are the means \pm SD from five independent experiments (A \& B). NS: not significant. (C) Apo-MPs, harvested from apoptotic ATRA-NB4 cell cultures (4 hours after UV irradiation), were either labeled (apo-MP*) or not labeled (apo-MP) with fluorescent FITC-conjugated CX3CL1 monoclonal antibody. Subsequently, NR8383 cells were incubated with either apo-MPs, apo-MP* or IgG (as control) before flow cytometry analysis. This is a representative picture from four independent experiments.

by its specific antibody $(p<0.05)$. We then demonstrate that exogenous CX3CL1 protein could enhance the transmigratory activity of NR8383 cells in a dose-dependent manner $(p<0.05)$, and this enhancement was also inhibited when CX3CR1 on the NR8383 cells were previously blocked by its specific antibody before treatment with exogenous CX3CL1 (Fig. 5A).

Next, the question whether apo-MPs, released by apoptotic ATRA-NB4 cells, have pro-transmigratory activity relative to NR8383 cells was explored. To do this, apo-MPs were incubated with the NR8383 cells before transmigratory assays. Figure 5B shows that NR8383 cells incubated with apo-MPs, derived from apoptotic ATRA-NB4 cells, had a significantly higher transmigratory activity than those cells incubated with either vehicle 
alone or MPs derived from UV-untreated ATRA-NB4 cells ( $p<0.05$ and $p<0.05$; respectively); and this pro-transmigratory activity could be significantly inhibited by blocking the surface CX3CL1 of apo-MPs with its specific antibody before incubating these apo-MPs with NR8383 cells $(p<0.001)$. Finally, apo-MPs were labeled with a fluorescent FITC-conjugated CX3CL1 monoclonal antibody before being incubated with NR8383 cells for flow cytometry analysis. Figure 5C demonstrates that the fluorescent FITC-labeled conjugates were able to be transported from the labeled apo-MPs to the surface of recipient NR8383 cells, indicating that apo-MPs are able to adhere and/or fuse to the cell membrane of the recipient NR8383 cells.

\section{Discussion}

Release of "find-me" signals by apoptotic cells is crucial to the docking of macrophages with apoptotic cells for subsequent phagocytosis during the final stage of the apoptosis program [19]. The present study demonstrates CX3CL1(+) apo-MPs, released by apoptotic APL cells, contribute to the chemoattraction of $\operatorname{AM} \phi$ toward apoptotic cells.

We demonstrate that the release of CX3CL1(+) apo-MPs and CX3CL1 by apoptotic ATRANB4 cells was enhanced during the early apoptotic process, and this was accompanied with a simultaneous decrease in the surface expression of CX3CL1 on the apoptotic ATRA-NB4 cells. To confirm the crucial role of CX3CL1-CX3CR1 axis, our data indicate that CX3CL1enhances the transmigration of NR8383 cells toward apoptotic cells, and this is dependent on the presence of CX3CR1 on the NR8383 cells (Fig. 1,2,5).

Release of MPs is a well-known characteristic of cells undergoing apoptosis, and these MPs are involved in the chemoattraction of mononuclear phagocytes to apoptotic cells [7]. To extend this finding, we demonstrated that an increased amount of CX3CL1(+) apo-MPs were released by apoptotic ATRA-NB4 cells to enhance the transmigration of NR8383 cells toward apoptotic cells. We confirmed that apo-MPs released during the apoptotic process differ phenotypically and quantitatively from those MPs released during cellular activation [20,21]. Recent studies indicate that MPs released by activated neutrophils or differentiated ATRAAPL cells are associated with anti-inflammatory property in which the anti-inflammatory mediator annexin A1 is involved [15, 16, 22]. In contrast to these studies, we demonstrate that apo-MPs released by apoptotic ATRA-NB4 cells induced a higher pro-transmigratory activity on NR8383 cells than those MPs released by living ATRA-NB4 cells (Fig. 5), and this was in parallel with a higher amount of CX3CL1(+) MPs released by apoptotic ATRA-NB4 cells than released by the living cells. CX3CL1 on the surface of apo-MPs is likely to act as an adhesion molecule to promote the adhesion of apo-MPs to recipient NR8383 cells via the CX3CL1-CX3CR1 axis (Fig. 5C) [23], this may result in the release of a larger amount of CX3CL1 from apo-MPs to the surface of NR8383 cells to build up a chemotactic gradient in navigating NR8383 cells toward apoptotic ATRA-NB4 cells. To further support the crucial role of CX3CL1 in the CX3CL1(+)MPs, our results demonstrate that pro-transmigratory property of apo-MPs released by apoptotic ATRA-NB4 cells was inhibited by blocking their surface CX3CL1 with its specific antibody (Fig. 5). Taken together, our results indicate that CX3CL1 mediates the pro-transmigratory property of the CX3CL1(+) apo-MPs released by apoptotic ATRA-NB4 cells in the transmigration of NR8383 cells toward apoptotic ATRANB4 cells.

CX3CL1 released by apoptotic cells is also able to promote the macrophage's capacity in engulfing apoptotic cells. In this context, CX3CL1 enhances the binding of macrophages to apoptotic cells through up-regulating MFG-E8 (milk fat globule epidermal growth factor 8 ), prevents macrophages from apoptosis and enhances monocyte recruitment into alveolar space to expand the AM $\phi$ pool [24-28]. On the other hand, we also demonstrate that, when ATRA-NB4 cells progressed from early to late apoptotic stage, the surface expression of CX3CL1 was increased (Fig. 4), which may act as an adhesion molecule to promote the binding of those apoptotic cells to NR8383 cells, via CX3CL1-CX3CR1 axis, for subsequent 
phagocytosis, and this will prevent late apoptotic cells from releasing toxic molecules to cause further tissue damage. In agreement with a previous study [9], we also demonstrated that total cellular CX3CL1 protein level was persistently elevated in the apoptotic ATRANB4 cells (Fig. 4B), indicating that CX3CL1 protein production is enhanced to expand the cytoplasmic pool after induction of the apoptotic process. The cytoplasmic CX3CL1 is likely to be mobilized to plasma membrane to expand the surface CX3CL1 in apoptotic ATRA-NB4 cells after induction of apoptotic process because neither the level of total cellular CX3CL1 protein nor the level of CX3CL1 or CX3CL1(+) apo-MPs in the CM had a significant change during the later stage of apoptotic process (Fig. 2,3). However, this warrants further study.

Previous studies in DS have focused on investigating the role of cytokines in the pathogenesis of acute lung injury during acute inflammatory phase. In this context, IL-8, MCP-1, IL-1 $\beta$ and GRO- $\alpha$ play an important role in inducing transmigration of differentiated APL cells from blood stream into alveolar space [1-3, 29, 30]. In contrast to these studies, we studied the mechanism underlying the clearance of apoptotic APL cells during resolution phase in DS patients. Based on our results, we postulate that apoptotic ATRA-APL cells are likely to release CX3CL1(+) MPs and CX3CL1 to enhance transmigration of AM $\phi$ toward apoptotic cells allowing subsequent phagocytosis in the alveolar spaces in DS patients with acute lung injury. However, this warrants further study to determine the level of CX3CL1(+) MPs and CX3CL1 in the bronchioalveolar lavage in patients with DS or in the animal DS model. In addition, we have reported previously that, after interacting with apoptotic cells, AM $\phi$ release lipoxin $\mathrm{A} 4$ to enhance the phagocytic activity of $\mathrm{AM} \phi$, promote monocyte recruitment and inhibit neutrophils trafficking into alveolar space [26, 28], this may further contribute to the clearance of apoptotic cells from alveolar spaces during the resolution phase in DS patients. Therefore, we conclude that CX3CL1, released by apoptotic APL cells, plays a crucial role in the clearance of apoptotic APL cells from alveolar space during resolution phase in DS patients. Our work also provides a rationale for further clinical studies aimed at defining the pathophysiological role of CX3CL1 in the resolution of acute lung injury caused by other etiologies.

\section{Acknowledgments}

This study was conduct in the Clinical Research Core Laboratory of Taipei Veterans General Hospital. We are indebted to Dr. Ralph Kirby, Department of Life Sciences, National Yang-Ming University, and Charles Hsu, Department of Biology, Massachusetts Institute of Technology, USA, for their help with the language editing.

This study was supported by National Science Council, Taiwan; (NSC-101-2314-B-010 -026) and Taipei Veterans General Hospital; (V102C-050)

\section{References}

-1 Frankel SR, Eardley A, Lauwers G, Weiss M, Warrell RP Jr: The "retinoic acid syndrome" in acute promyelocytic leukemia. Ann Intern Med 1992;117:292-296.

-2 Tsai WH, Hsu HC, Lin CC, Ho CK, Kou YR: Role of interleukin-8 and growth-regulated oncogene-alpha in the chemotactic migration of all-trans retinoic acid-treated promyelocytic leukemic cells toward alveolar epithelial cells. Crit Care Med 2007;35:879-885.

3 Tsai WH, Shih CH, Lin CC, Ho CK, Hsu FC, Hsu HC: Monocyte chemotactic protein-1 in the migration of differentiated leukaemic cells toward alveolar epithelial cells. Eur Respir J 2008;31:957-962.

-4 Tsai WH, Shih CH, Wu HY, Chien HY, Chiang YC, Lai SL, Hsu SC, Kou YR, Hsu HC: Role of lipoxin a4 in the cellto-cell interaction between all-trans retinoic acid-treated acute promyelocytic leukemic cells and alveolar macrophages. J Cell Physiol 2012;227:1123-1129.

Rubins JB: Alveolar macrophages. Am J Resp Crit Care 2003;167:103-104.

Knies UE, Behrensdorf HA, Mitchell CA, Deutsch U, Risau W, Drexler HCA, Clauss M: Regulation of endothelial monocyte-activating polypeptide ii release by apoptosis. Proc Natl Acad Sci USA 1998;95:12322-12327. 
Tsai et al.: CX3CL1(+) Microparticles Stimulate Alveolar M $\phi$ Chemotaxis

7 Lauber K, Bohn E, Krober SM, Xiao YJ, Blumenthal SG, Lindemann RK, Marini P, Wiedig C, Zobywalski A, Baksh S, Xu Y, Autenrieth IB, Schulze-Osthoff K, Belka C, Stuhler G, Wesselborg S: Apoptotic cells induce migration of phagocytes via caspase-3-mediated release of a lipid attraction signal. Cell 2003;113:717-730.

8 Segundo C, Medina F, Rodriguez C, Martinez-Palencia R, Leyva-Cobian F, Brieva JA: Surface molecule loss and bleb formation by human germinal center b cells undergoing apoptosis: Role of apoptotic blebs in monocyte chemotaxis. Blood 1999;94:1012-1020.

-9 Truman LA, Ford CA, Pasikowska M, Pound JD, Wilkinson SJ, Dumitriu IE, Melville L, Melrose LA, Ogden CA, Nibbs R, Graham G, Combadiere C, Gregory CD: Cx3cl1/fractalkine is released from apoptotic lymphocytes to stimulate macrophage chemotaxis. Blood 2008;112:5026-5036.

10 Bazan JF, Bacon KB, Hardiman G, Wang W, Soo K, Rossi D, Greaves DR, Zlotnik A, Schall TJ: A new class of membrane-bound chemokine with a cx3c motif. Nature 1997;385:640-644.

$\checkmark 11$ Helmke RJ, German VF, Mangos JA: A continuous alveolar macrophage cell line: Comparisons with freshly derived alveolar macrophages. In Vitro Cell Dev Biol 1989;25:44-48.

12 Esmann L, Idel C, Sarkar A, Hellberg L, Behnen M, Möller S, van Zandbergen G, Klinger M, Köhl J, Bussmeyer U, Solbach W, Laskay T: Phagocytosis of apoptotic cells by neutrophil granulocytes: Diminished proinflammatory neutrophil functions in the presence of apoptotic cells. J Immunol 2010;184:391-400.

13 Hasper HJ, Weghorst RM, Richel DJ, Meerwaldt JH, Olthuis FMFG, Schenkeveld CEI: A new four-color flow cytometric assay to detect apoptosis in lymphocyte subsets of cultured peripheral blood cells. Cytometry 2000;40:167-171.

14 Gasser O, Hess C, Miot S, Deon C, Sanchez J-C, Schifferli JüA: Characterisation and properties of ectosomes released by human polymorphonuclear neutrophils. Exp Cell Res 2003;285:243-257.

15 Tsai WH, Chien HY, Shih CH, Lai SL, Li IT, Hsu SC, Kou YR, Hsu HC: Annexin a1 mediates the antiinflammatory effects during the granulocytic differentiation process in all-trans retinoic acid-treated acute promyelocytic leukemic cells. J Cell Physiol 2012;227:3661-3669.

-16 Dalli J, Norling LV, Renshaw D, Cooper D, Leung KY, Perretti M: Annexin 1 mediates the rapid antiinflammatory effects of neutrophil-derived microparticles. Blood 2008;112:2512-2519.

17 Tsai WH, Shih CH, Yu YB, Hsu HC: Plasma levels in sepsis patients of annexin a1, lipoxin a4, macrophage inflammatory protein-3a, and neutrophil gelatinase-associated lipocalin. J Chin Med Assoc 2013;76:486490.

18 Shih CH, Tsai WH, Huang SW, Chu JS, Hsu SC, Hsu HC: "Effects of high concentration oxygen treatment on traumatic pneumothorax in adult rabbits". Chin J Physiol 2012;55:178-183.

19 Grimsley C, Ravichandran KS: Cues for apoptotic cell engulfment: Eat-me, don't eat-me and come-get-me signals. Trends Cell Biol 2003;13:648-656.

20 Jimenez JJ, Jy W, Mauro LM, Soderland C, Horstman LL, Ahn YS: Endothelial cells release phenotypically and quantitatively distinct microparticles in activation and apoptosis. Thromb Res 2003;109:175-180.

21 Mause SF, Weber C: Microparticles: Protagonists of a novel communication network for intercellular information exchange. Circ Res 2010;107:1047-1057.

-22 Gasser 0, Schifferli JA: Activated polymorphonuclear neutrophils disseminate anti-inflammatory microparticles by ectocytosis. Blood 2004;104:2543-2548.

23 Mack M, Kleinschmidt A, Bruhl H, Klier C, Nelson PJ, Cihak J, Plachy J, Stangassinger M, Erfle V, Schlondorff D: Transfer of the chemokine receptor ccr5 between cells by membrane-derived microparticles: A mechanism for cellular human immunodeficiency virus 1 infection. Nature Med 2000;6:769-775.

-24 Akakura S, Singh S, Spataro M, Akakura R, Kim JI, Albert ML, Birge RB: The opsonin mfg-e8 is a ligand for the alphavbeta5 integrin and triggers dock180-dependent rac1 activation for the phagocytosis of apoptotic cells. Exp Cell Res 2004;292:403-416.

-25 Miksa M, Amin D, Wu R, Ravikumar TS, Wang P: Fractalkine-induced mfg-e8 leads to enhanced apoptotic cell clearance by macrophages. Mol Med 2007;13:553-560.

-26 Serhan CN, Savill J: Resolution of inflammation: The beginning programs the end. Nat Immunol 2005;6:1191-1197.

27 Ancuta P, Rao R, Moses A, Mehle A, Shaw SK, Luscinskas FW, Gabuzda D: Fractalkine preferentially mediates arrest and migration of cd16+ monocytes. J Exp Med 2003;197:1701-1707.

-28 Maddox JF, Serhan CN: Lipoxin a4 and b4 are potent stimuli for human monocyte migration and adhesion: Selective inactivation by dehydrogenation and reduction. J Exp Med 1996;183:137-146.

-29 Luesink M, Jansen JH: Advances in understanding the pulmonary infiltration in acute promyelocytic leukaemia. Br J Haematol 2010;151:209-220.

-30 Hsu H-C, Tsai W-H, Chen P-G, Hsu M-L, Ho C-K, Wang S-Y: In vitro effect of granulocyte-colony stimulating factor and all-trans retinoic acid on the expression of inflammatory cytokines and adhesion molecules in acute promyelocytic leukemic cells. Eu J Haematol 1999;63:11-18. 